\title{
Optical Nanoantennas for Multiband Surface-Enhanced Infrared and Raman Spectroscopy
}

Cristiano D'Andrea, ${ }^{\dagger, \nabla}$ Jörg Bochterle, ${ }^{\neq, \nabla}$ Andrea Toma, ${ }^{\S}$ Christian Huck, ${ }^{\ddagger}$ Frank Neubrech, ${ }^{\neq, \perp}$ Elena Messina, $^{\dagger}$ Barbara Fazio, ${ }^{\dagger}$ Onofrio M. Maragò, ${ }^{\dagger}$ Enzo Di Fabrizio,,$" \#$ Marc Lamy de La Chapelle, ${ }^{\triangle}$ Pietro G. Gucciardi, ${ }^{, *}$ and Annemarie Pucci ${ }^{\ddagger}$ *

${ }^{\dagger}$ CNR IPCF Istituto per i Processi Chimico-Fisici, Viale F. Stagno D'Alcontres 37, I-98156, Messina, Italy, ${ }^{\ddagger}$ Kirchhoff-Institute for Physics, Heidelberg University, Im Neuenheimer Feld 227, 69120 Heidelberg, Germany, ${ }^{\S}$ Nanostructures, Istituto Italiano di Tecnologia, Via Morego 30, 16163 Genova, Italy, ${ }^{\perp}$ 4th Physics Institute, University of Stuttgart, Pfaffenwaldring 57, 70569 Stuttgart, Germany, "Physical Science and Engineering and BESE Divisions, KAUST, King Abdullah University of Science and Technology, Thuwal, 23955-6900, Kingdom of Saudi Arabia, "BIONEM Lab, University of Magna Graecia, Campus Salvatore Venuta, Viale Europa 88100, Germaneto-Catanzaro, Italy, and ${ }^{\triangle}$ Laboratoire CSPBAT, UMR 7244, UFR SMBH, Université Paris 13, 74 Rue Marcel Cachin, 93017 Bobigny, France. ${ }^{\nabla}$ C. D'Andrea and J. Bochterle contributed equally to this work.

\begin{abstract}
In this article we show that linear nanoantennas can be used as shared substrates for surface-enhanced Raman and infrared spectroscopy (SERS and SEIRS, respectively). This is done by engineering the plasmonic properties of the nanoantennas, so to make them resonant in both the visible (transversal resonance) and the infrared (longitudinal resonance), and by rotating the excitation field polarization to selectively take advantage of each resonance and achieve SERS and SEIRS on the same nanoantennas. As a proof of concept, we have fabricated gold nanoantennas by electron beam lithography on calcium
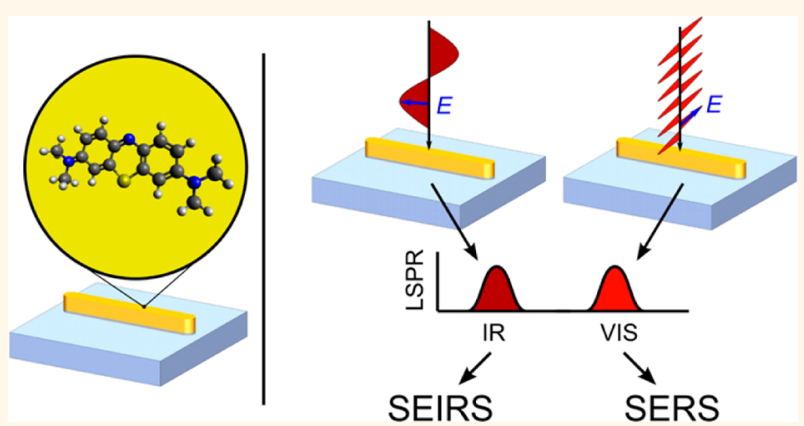

difluoride (1-2 $\mu \mathrm{m}$ long, $60 \mathrm{~nm}$ wide, $60 \mathrm{~nm}$ high) that exhibit a transverse plasmonic resonance in the visible (640 nm) and a particularly strong longitudinal dipolar resonance in the infrared (tunable in the $1280-3100 \mathrm{~cm}^{-1}$ energy range as a function of the length). SERS and SEIRS detection of methylene blue molecules adsorbed on the nanoantenna's surface is accomplished, with signal enhancement factors of $5 \times 10^{2}$ for SERS (electromagnetic enhancement) and up to $10^{5}$ for SEIRS. Notably, we find that the field enhancement provided by the transverse resonance is sufficient to achieve SERS from single nanoantennas. Furthermore, we show that by properly tuning the nanoantenna length the signals of a multitude of vibrational modes can be enhanced with SEIRS. This simple concept of plasmonic nanosensor is highly suitable for integration on lab-on-a-chip schemes for label-free chemical and biomolecular identification with optimized performances.
\end{abstract}

KEYWORDS: surface-enhanced Raman spectroscopy (SERS) - surface-enhanced infrared spectroscopy (SEIRS) surface-enhanced infrared absorption (SEIRA) - optical nanoantenna $\cdot$ plasmonics $\cdot$ methylene blue $\cdot$ spectroscopy $\cdot$ nanosensor

$\mathrm{O}$ ptical nanoantennas ${ }^{1,2}$ convert freely propagating optical radiation into enhanced optical fields localized on nanometric regions of their surface, the so-called hot spots. ${ }^{3-9}$ Such an effect arises from the resonant coupling of light with collective oscillations of conduction electrons of metal nanoparticles called localized surface plasmon resonances (LSPR). ${ }^{10,11}$ The huge electromagnetic field enhancement at the hot spots enables a dramatic increase of the vibrational signal of molecules located therein. Surface-enhanced Raman spectroscopy (SERS), ${ }^{12-14}$ in which Raman scattering is magnified by factors between $10^{2}$ and $10^{8}$ (see ref 15 ), is probably the most recognized example of plasmon-enhanced spectroscopy, allowing one to carry out vibrational analysis with excitation at optical frequencies down to the single-molecule level. ${ }^{16,17}$ Plasmonic nanoantennas can also be used to amplify the infrared (IR) vibrational signals of adsorbed molecules and thin layers. Enhancement factors (EF) of up to $10^{4}$ have been demonstrated, ${ }^{18-24}$ increasing to $10^{5}$ when the LSPR of a nanoantenna is resonantly tuned to match the IR vibration energy of the probe molecule. ${ }^{25,26}$ The latter

\section{* Address correspondence to gucciardi@me.cnr.it, pucci@kip.uni-heidelberg.de.}

Received for review January 29, 2013 and accepted March 26, 2013.

Published online

10.1021/nn4004764

C XXXX American Chemical Society 

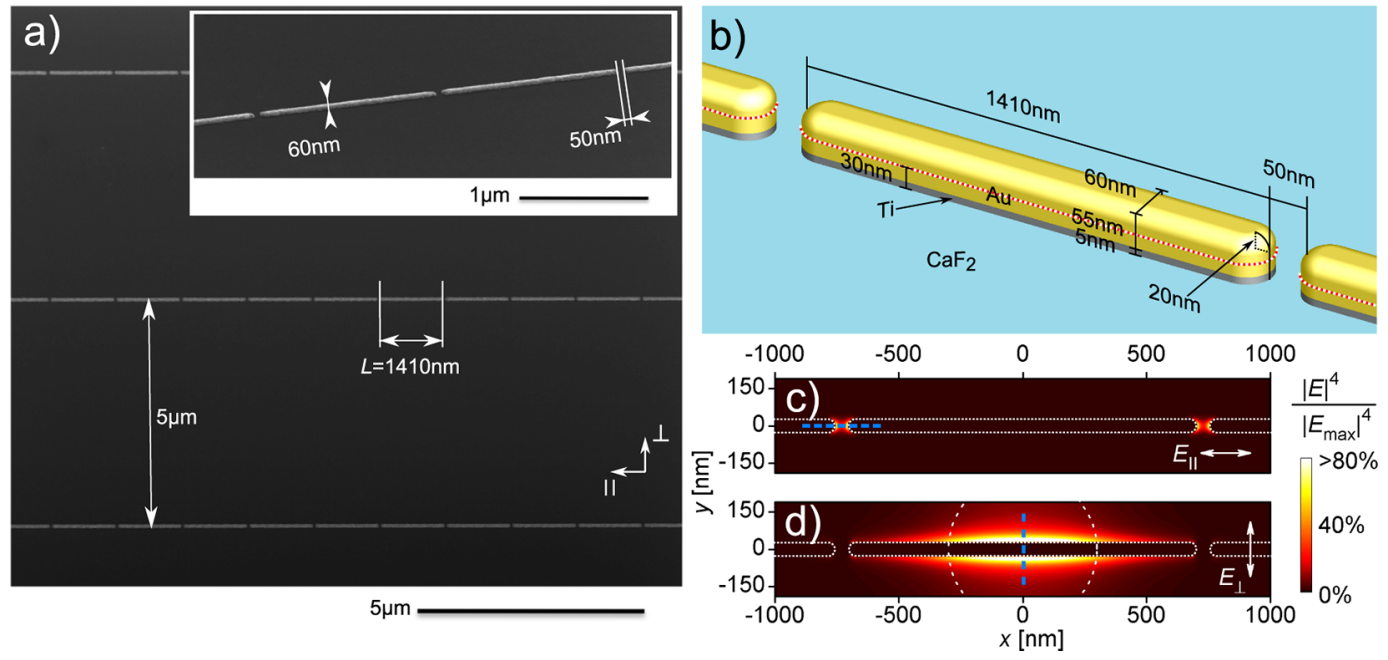

Figure 1. (a) SEM image of the gold nanoantennas arrangement on the $\mathrm{CaF}_{2}$ substrate. The inset shows a zoom-in under an oblique view. The symbols $\|$ and $\perp$ indicate the longitudinal and the transverse directions, respectively, with respect to the nanoantenna's long axis. (b) Schematic sketch of the antenna geometry used for theoretical calculations. (c, d) Maps of squared near-field intensity calculated in a plane $30 \mathrm{~nm}$ above the $\mathrm{CaF}_{2}$ surface (intersectional lines shown as dotted line in (b)) at energies corresponding to resonances in the near-field of the fundamental longitudinal dipolar mode in the IR (c, field $E_{\|}$polarized parallel to the long axis, $1751 \mathrm{~cm}^{-1}$ ) and the laser excitation used to excite the transverse plasmon resonance mode (d, field $E_{\perp}$ parallel to the nanoantenna short axis, $633 \mathrm{~nm}$ ). The fields in (d) are normalized to the value of the highest field $E_{\max }$ (see Supporting Information). The incident field is simulated using a plane wave in the IR (c) and a Gaussian beam (d) in the visible (the dashed circle indicates the area of the laser spot where $E / E_{0} \geq 1 / e$ with a diameter of about $600 \mathrm{~nm}$ ). The contours of the antennas are indicated by the dotted lines. The blue lines indicate cross sections shown in Figure S2 (see Supporting Information for more details).

effect is named surface-enhanced infrared scattering (SEIRS) because, for resonant nanoantennas in the IR, light scattering (proportional to the fourth power of the electric near-field) ${ }^{27}$ dominates the extinction signal and the molecular vibrations show up as Fano-type signals superimposed on the resonant plasmonic background of the nanoantenna (see ref 28 for more details). SEIRS with plasmonic light scattering is becoming an increasingly important technique as a complementary tool to SERS with similarly high vibrational sensivity. ${ }^{28-30}$ Because of the different excitation mechanism, different selection rules and different cross sections hold for IR and Raman activity. Complete vibrational information can be obtained, therefore, only with simultaneous application of both techniques. $^{31-33}$

SERS, SEIRS, and surface-enhanced IR absorption (SEIRA) have been applied for chemical, biological, and pharmaceutical analysis and sensing ${ }^{34-40}$ using specific plasmonic nanostructures to enhance either the IR or the Raman signals. Some approaches have been reported on the development of shared substrates for SERS and SEIRS sensing, ${ }^{41,42}$ indeed more versatile and simple to integrate in a lab-on-a-chip architecture. Examples include arrays of gold nanoshells, ${ }^{19,42}$ self-assembled microemulsions, self-assembled pulsed laser deposited gold nanoparticles clusters, ${ }^{43-45}$ sputtered thin-film gold electrodes, ${ }^{46}$ near-field coupled silver nanowires, ${ }^{47}$ electrochemically treated copper surfaces, ${ }^{48}$ and silver-nanoparticles clusters. ${ }^{49}$ These nanostructures feature broad
LSPRs from the visible to the IR, thus being effective for plasmon-enhanced Raman and IR spectroscopy. To optimize the response of a combined SERS-SEIRS sensor, however, optical nanoantennas with engineered shapes and dimensions are much more desirable. Linear nanoantennas, featuring two distinct LSPRs, allow one to precisely tune the first LSPR to specific IR spectral regions, ${ }^{50-52}$ so to selectively enhance the desired IR vibrations, ${ }^{25}$ and to adjust the second LSPR in the visible, close to the laser excitation, for maximum SERS enhancement. 53,54

Here we show that linear gold nanoantennas made by electron beam lithography $(\mathrm{EBL})$ on $\mathrm{CaF}_{2}$ represent a viable platform for simultaneous SERS-SEIRS molecular analysis. We prove that, exploiting the longitudinal dipolar resonance (in the IR for 1-2 $\mu \mathrm{m}$ long antennas ${ }^{52}$ ), it is possible to achieve SEIRS, while the transverse plasmon resonance (in the red spectral region for $60 \mathrm{~nm}$ wide and high antennas) can be used for SERS with laser excitation in the visible. ${ }^{55}$ By simply switching the polarization of the excitation fields, both SERS and SEIRS molecular analysis can be obtained on the same sample architecture.

\section{RESULTS AND DISCUSSION}

Optical Nanoantennas. Nanoantennas have been fabricated by electron beam lithography on $\mathrm{CaF}_{2}$ (100) substrates (see Methods). Due to the insulating nature of the substrate, a bilayer of resist and aluminum, $10 \mathrm{~nm}$ thick, was used to prevent charging of the sample. After aluminum removal and resist development, 
TABLE 1. Selection of MB Vibrational Frequencies (in $\left.\mathrm{cm}^{-1}\right)^{a}$

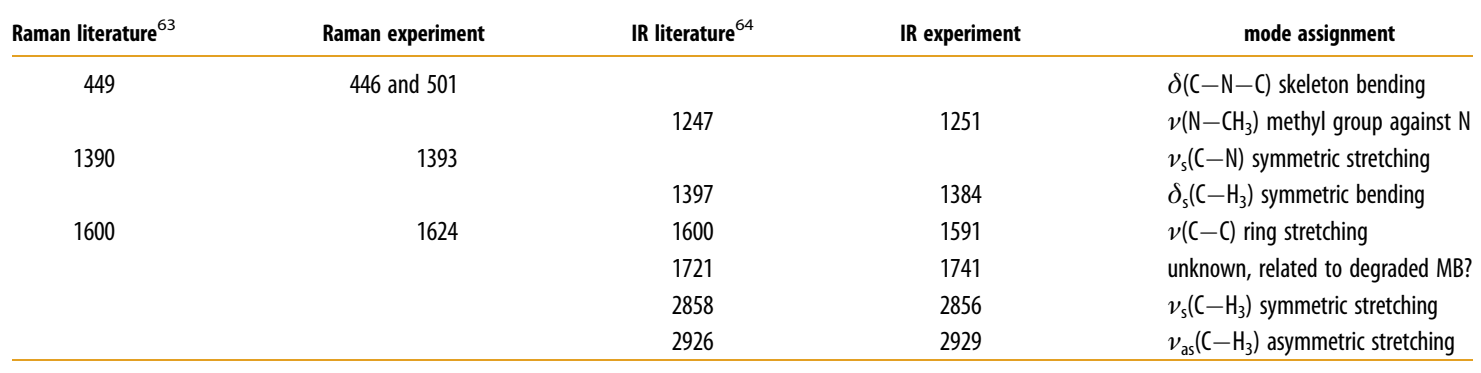

${ }^{a}$ Measured values of this work compared to the literature.

a combined evaporation of gold and lift-off was the last step to fabricate the nanoantennas. Scanning electron microscopy images are shown in Figure 1a. We have investigated four sets of antenna arrays fabricated on the same $\mathrm{CaF}_{2}$ wafer, each one containing nanoantennas of a specific length $(L=1910,1410,1110$, and $710 \mathrm{~nm})$. Widths $(w=60 \mathrm{~nm})$ and heights $(h=60 \mathrm{~nm}$, including $5 \mathrm{~nm}$ of Ti adhesion layer) are the same for all the antennas. Within each array, the nanoantennas are arranged in a tip-to-tip configuration with gaps of $50 \mathrm{~nm}$ (see inset). Each chain is separated from the neighboring one by $5 \mu \mathrm{m}$. With this spacing, conditions for transverse coupling in the IR are optimal and field enhancement at the antenna apex is not lowered by transverse near-field coupling. ${ }^{52}$ The longitudinal gap of $50 \mathrm{~nm}$ increases the antenna density within the array. Its small size increases IR near-field enhancement slightly, but is not important for the horizontal plasmon resonance in the visible. Thus, the reported principle is also valid for single antennas and as such can be exploited in a combination of a Raman and an IR laser setup.

To prove our concept, we have used methylene blue (MB) as probe molecule $\left(\mathrm{C}_{16} \mathrm{H}_{18} \mathrm{~N}_{3} \mathrm{SCl}\right.$; see inset of Figure S1a in the Supporting Information for the chemical structure drawing). MB is a heterocyclic aromatic compound with many applications in biosensing, ${ }^{56}$ photodynamic therapies, ${ }^{57}$ and fundamental SERS experiments. ${ }^{51,58,59}$ Like various other dyes, the electronic resonances of $\mathrm{MB}^{60,61}$ (absorption spectra are displayed in Figure S1a) permit exploiting the enhancement due to resonant Raman scattering (RRS) effects to record spectra of molecules adsorbed on substrates with low or no plasmonic enhancement. ${ }^{59,62}$ This allows us to carry out reference RRS measurements and compare them with SERS, in order to precisely evaluate the enhancement factor. The most characteristic vibrational modes of $\mathrm{MB}$ are listed in Table 1. Intense Raman and IR active vibrations occur in the $400-1650 \mathrm{~cm}^{-1}$ range. The strongly IR active $\mathrm{C}-\mathrm{H}$ stretching modes from $\mathrm{CH}_{3}$ groups show up at higher energies $\left(2850-2950 \mathrm{~cm}^{-1}\right)$. MB has been bound to gold surfaces following standard procedures (see Methods).

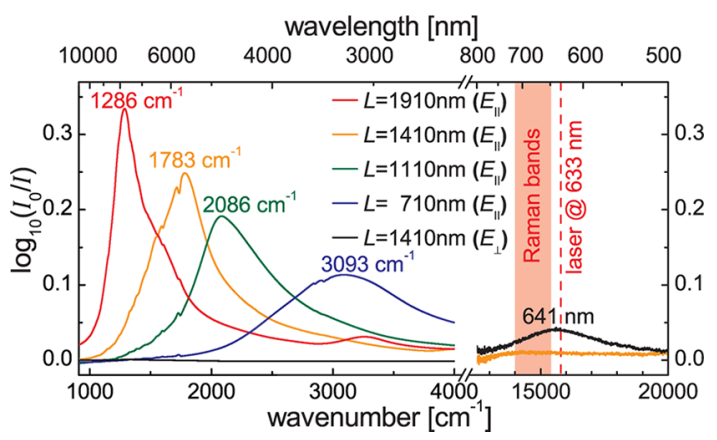

Figure 2. IR and visible extinction spectra of the four sets of nanoantennas after binding with methylene blue (red line: $L=1910 \mathrm{~nm}$, orange line: $L=1410 \mathrm{~nm}$, green line: $L=1110 \mathrm{~nm}$, blue line: $L=710 \mathrm{~nm}$ ). The absorbance value $\log _{10}\left(I_{0} / I\right)$ is reported, with $I$ and $I_{0}$ being the radiation intensities transmitted through the $\mathrm{CaF}_{2}$ substrate with and without the antennas, respectively. The spectra are acquired with light polarized parallel (longitudinal, $E_{\|}$) and perpendicular (transversal, $E_{\perp}$ ) to the nanoantennas, in order to excite respectively both the long axis and the short axis LSPRs. In the IR spectra the Fano-type features associated with the enhanced IR scattering of MB show up as distortions of the broad plasmonic absorption background of the nanoantennas. In the visible, the plasmonic resonance is independent of the antenna length, thus only the results of one set of antennas $(L=1410 \mathrm{~nm})$ are displayed. The red dashed line indicates the SERS excitation laser wavelength $(633 \mathrm{~nm})$. The red shaded area depicts the spectral position of the Raman bands (between 400 and $1700 \mathrm{~cm}^{-1}$ ) of the MB excited at $633 \mathrm{~nm}$.

Determination of LSPRs. Figure 2 displays the extinction spectra of the four sets of nanoantennas after adsorption of methylene blue. The IR LSPRs show up exciting the samples with a longitudinal field, $E_{\|}$, i.e., polarized along the long axis ${ }^{52,65}$ (experimental details in the Methods section). Resonances are found between 1280 and $3100 \mathrm{~cm}^{-1}$ (Figure 2, red, orange, green, and blue lines), matching the vibrational fingerprint modes of many molecular species. In the visible, a single LSPR peak at $641 \mathrm{~nm}$ (Figure 2, black line) is observed for the transversal excitation field, $E_{\perp}$, i.e., polarized parallel to the short axis (experimental details in the Methods section). Because of the large aspect ratio, the energy of this transverse LSPR resonance is independent of the antennas' lengths and corresponds to what is expected for a gold nanowire of about $60 \mathrm{~nm}$ in diameter. ${ }^{55,66}$ 
In the visible range, higher order multipolar resonances for longitudinally polarized excitation (Figure 2, orange line) have not been detected (in accord with refs 67 and 68). Our results on the plasmonic extinction underline that with such an easily tunable nanoantenna system both LSPR frequencies can be optimized independently, and thus field enhancement for SERS and SEIRS is possible on the same nanoantenna. Changing the antennas' length shifts the long axis LSPR to the desired vibrational frequency of the molecule, permitting optimization of the SEIRS enhancement. For maximum SERS, the width of the structures and the excitation laser wavelength have been chosen to have resonant coupling of both the excitation and the Raman bands to the short axis LSPR when using $633 \mathrm{~nm}$ laser excitation ${ }^{53,54}$ (see dashed line and shaded area in Figure 2).

The different spatial distributions of the enhanced fields in the visible and the IR have been studied by finite difference time domain (FDTD) numerical calculations (see Methods). The hot spots in the IR at the fundamental resonance are localized at the antenna apexes (Figure 1c) with a spatial extension of approximately the sectional area of the antenna, i.e., $2 \times w \times h$ $=7200 \mathrm{~nm}^{2}$. Molecules adsorbed on these sites dominate the total SEIRS signal; thus we use this area to calculate the enhancement factor later on. Conversely, when the transverse LSPR is resonantly excited in the visible range, the hot-spot region appears as a distributed zone (Figure 1d), extended along the laser spot $\left(d_{\text {beam }} \approx 600 \mathrm{~nm}\right)$ and confined close to the nanoantenna lateral surface. Accordingly, mainly the molecules adsorbed on the area $A_{\text {SERS }}=2 \times h \times d_{\text {beam }} \approx 7 \times 10^{4} \mathrm{~nm}^{2}$ contribute to the enhanced SERS signal.

SERS experiments have been carried out on individual nanoantennas, focusing a HeNe laser beam, resonant with the short axis LSPR $\left(\lambda_{\text {laser }}=633 \mathrm{~nm}\right)$, on a spot $\left(d_{\text {beam }} \approx 600 \mathrm{~nm}\right)$ at the center of the antenna (details in the Methods). At $633 \mathrm{~nm}$ mainly MB monomers and dimers are resonantly excited (Figure S1a). We therefore expect that most of the signal measured in our experiments is due to scattering from monomers/dimers and only to a smaller extent from aggregates deposited on the nanoantenna surface. Figure $3 a$ (orange line) shows the SERS spectrum obtained for transversal laser field polarization $\left(E_{\perp}\right)$ on a $L=1410 \mathrm{~nm}$ antenna, compared with the RRS signal (Figure 3a, hollow symbols) observed for polarization longitudinal to the nanoantenna $\left(E_{\|}\right)$. The SERS signal is $c a .100$ times more intense than the RRS. The SERS spectrum displays the major Raman active vibrations ${ }^{63,69}$ (listed in Table 1) with an $\mathrm{S} / \mathrm{N}$ ratio of $>20$ at a laser power as small as $500 \mathrm{nW}$ (integration time $30 \mathrm{~s}$ ), while a hundred times more power $(50 \mu \mathrm{W})$ is required to obtain a measurable RRS signal using longitudinally polarized light (same integration time). Notice that in the latter case the signal on the nanoantenna

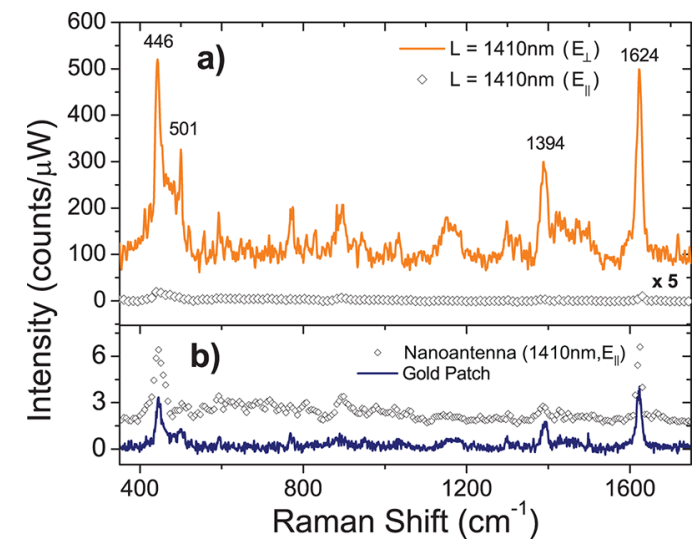

Figure 3. (a, orange line) SERS spectrum of methylene blue adsorbed on a single $L=1410 \mathrm{~nm}$ nanoantenna for laser field polarization $\left(E_{\perp}\right)$ parallel to the short axis $(633 \mathrm{~nm}$ laser, power $500 \mathrm{nW}$, integration time $30 \mathrm{~s}$ ). (a, hollow symbols) RRS signal acquired on the same antenna with polarization $\left(E_{\|}\right)$parallel to the long axis (633 nm laser, power $50 \mu \mathrm{W}$, integration time $30 \mathrm{~s}$ ). The upper (orange) spectrum is vertically shifted (by 100 counts) and the RRS intensity is multiplied by a factor of 5 , for clarity purposes. (b) Comparison of the RRS signal measured on the antenna with longitudinal laser polarization (hollow symbols) with the RRS of MB adsorbed on a flat gold patch (dark blue line) under the same experimental conditions (laser power $50 \mu \mathrm{W}$, integration time $30 \mathrm{~s}$ ). The upper spectrum is vertically shifted for clarity. All spectra are baseline corrected and normalized to the laser power.

(Figure 3b, hollow symbols) is comparable with both the RRS signal (Figure 3b, blue line) measured on the flat gold patches present on the same sample (on which $\mathrm{MB}$ is similarly bound) and the signal measured on the $\mathrm{CaF}_{2}$ substrate far from the antennas (spectrum not shown). This observation suggests that (i) for longitudinal polarization, no plasmonic enhancement takes place at $633 \mathrm{~nm}$, i.e., higher order LSPR modes are not playing any significant role in amplifying the Raman signal; (ii) the RRS signal measured on the nanoantennas is almost originating from $\mathrm{MB}$ on the $\mathrm{CaF}_{2}$ substrate; and (iii) the contribution of $\mathrm{MB}$ on $\mathrm{CaF}_{2}$ to the total SERS signal (measured for transverse polarization) is only $1 \%$. Analogous to what is found for the extinction, the SERS intensity is independent of the nanoantenna length. No additional signal enhancement is observed when placing the laser spot in the gap region between two adjacent nanoantennas, independently of the field polarization, indicating negligible near-field coupling in gaps with sizes of $G_{x}=50 \mathrm{~nm}$, as expected. ${ }^{3}$ Antenna coupling certainly is not relevant in the vertical direction where the gap among the antennas is much larger (gap $G_{y}=5 \mu \mathrm{m}$, Figure 1a). To calculate the enhancement factor, $\mathrm{EF}$, we take the ratio between the SERS intensity of the $476 \mathrm{~cm}^{-1}$ peak (measured on the nanoantenna for transverse polarization) and the corresponding reference RRS signal (measured on the flat gold patch reference sample; see Methods), normalizing each signal to the number of probed molecules ( $N_{\text {SERS }}$ and $N_{\text {RRS }}$ ), the effective laser power 
$\left(P_{\mathrm{SERS}}\right.$ and $\left.P_{\mathrm{RRS}}\right)$, and the integration times ( $t_{\mathrm{SERS}}$ and $\left.t_{\mathrm{RRS}}\right)$. To account for the different number of probed molecules, we assume an equal molecular coverage (equal number of molecules per unit area) on both the nanoantenna and the flat gold patch. This is reasonable, since both structures are on the same chip and undergo the same immersion conditions (time and concentration) in the MB solution. With this assumption, and neglecting the very small contribution from $\mathrm{MB}$ on $\mathrm{CaF}_{2}$, the number of probed molecules is proportional to the surface within the effective excitation field in each of the two cases (i.e., $N_{\text {SERS }} \propto A_{\text {SERS }}$ and $N_{\text {RRS }} \propto A_{\text {RRS }}$ ). On the gold patch, the molecules that contribute are those within the laser spot area $A_{\mathrm{RRS}}=$ $\pi d_{\text {beam }}^{2} / 4$. On the nanoantennas, the MB molecules contributing to the SERS are those bound to the lateral area of the nanoantenna $A_{\text {SERS }}$ within the hot spot (Figure 1d). We find that at $633 \mathrm{~nm}$ the electromagnetic enhancement factor is $E F \approx 5 \times 10^{2}$. Similar values are observed for all the vibrational peaks. In order to check for the excitation wavelength that maximizes SERS, $\lambda_{\mathrm{SERS}}$ and to look for possible shifts between $\lambda_{\mathrm{SERS}}^{\max }$ and the visible range LSPR peak wavelength, ${ }^{54}$ further Raman experiments have been carried out exciting at $515 \mathrm{~nm}$ (Argon ion laser) and $785 \mathrm{~nm}$ (laser diode), i.e., using an excitation energy blue- and red-shifted with respect to the LSPR. We find that at $515 \mathrm{~nm}$, the nanoantenna induces a signal amplification of a factor $\sim 5$ with respect to the gold patch (Figure S3a and d in the Supporting Information), whereas no signal amplification is observed at $785 \mathrm{~nm}$ (Figure S3c and f). This kind of observation should be expected, ${ }^{53}$ since at $515 \mathrm{~nm}$ only the higher energy Raman bands fall in the tail of the LSPR, while at $785 \mathrm{~nm}$ both the laser excitation and the Raman bands are totally out of resonance. Maximum SERS is observed at $\lambda_{\mathrm{SERS}}^{\max }=$ $633 \mathrm{~nm}$, where both the laser and the Raman bands fall within the broad transverse LSPR of the nanoantenna. No relevant energy shift between the plasmonic properties in the near-field and the far-field ${ }^{70}$ (probed respectively by SERS and extinction spectroscopy) is observed in our nanoantennas. Much stronger EF can be obtained using various other kinds of metal nanoantennas. ${ }^{67}$ The discrepancy with respect to our finding is probably related to the weak near-field confinement and small dipole moment of the transverse LSPR of a $60 \mathrm{~nm}$ thin and very long nanowire. On the other hand, it should be noticed that, with the normalization procedure used in this work, the EF clearly includes only the electromagnetic enhancement and does not take into account the chemical enhancement, typically providing 1 or 2 orders of magnitude of additional signal amplification. In this case, at $633 \mathrm{~nm}$, we can expect a total EF between $10^{4}$ and $10^{5}$, i.e., comparable to what is reached with similar structures. ${ }^{67}$ To exclude systematic errors due to the normalization procedure, we have cross-checked the
Raman signal measured on flat gold with the Raman signal measured in liquid solutions ${ }^{53}$ (details in the Supporting Information).

SEIRS measurements have been carried out on the same sample used for SERS. The IR radiation was polarized parallel to the nanoantenna long axis $\left(E_{\|}\right)$in order to excite the longitudinal LSPR, shown in Figure 2. A zoom-in of the IR LSPRs (Figure $4 a$, b) reveals that the resonance profiles are slightly distorted: Fano-type signals are found at positions of various IR active vibrational frequencies of MB. The vibrational frequencies correspond to lines in IR reflection absorption spectra (IRRAS) of a MB monolayer prepared on a flat gold mirror from the same source material with the same procedure as used for SEIRS and SERS. An absorbance spectrum derived from IRRAS of MB on planar gold is shown in Figure 4c, where selected vibrational frequencies are marked with dashed lines (see Table 1). The peak at $1741 \mathrm{~cm}^{-1}$ is not reported in the literature. It may indicate degradation of MB due to storage under ambient conditions. ${ }^{64}$ As can be seen in Figure 4a and e, this peak strongly shows up in SEIRS due to the good match of its frequency to the LSPR. Notably, this peak does not seem to be Raman active.

With the asymmetry of the Fano-line itself being dependent on the relative positions of the plasmon resonance regarding the vibrational frequency, ${ }^{25,71}$ it is not straightforward to determine molecular parameters, for example, the precise vibrational wavenumber. In order to extract the vibrational oscillator parameter and EF from the tiny distortions of the IR LSPR spectrum, we calculated the second derivative of the spectra ${ }^{72}$ with respect to the photon wavenumber for the various antenna lengths (Figure $4 d$ to $g$ ). Due to the much larger width of the LSPR resonance, no baseline correction is necessary with such a procedure.

At the spectral positions of the MB vibrations, the second derivative deviates from zero. This deviation gets stronger if the vibrational frequency becomes closer to the spectral position of the LSPR. Accordingly, for transverse polarization with LSPR in the visible, no vibrational signal is observed in the IR. The peaks' size in the second derivative corresponds to the curvature of extinction around the vibrational frequency and is approximately proportional to the vibrational signal size (here the difference between minimum and maximum of the Fano-type vibrational contribution to extinction), independent of the asymmetry parameter, when the vibrational line width is constant. Since the width of one specific MB vibration for the same temperature and the same adsorption environment is a constant, we can directly compare the sizes of a peak in the second derivative for various antenna lengths. So, the peak size (and thus EF) is higher if the vibrational energy is closer to the LSPR peak. In order to quantitatively calculate the $\mathrm{EF}$, we fitted 

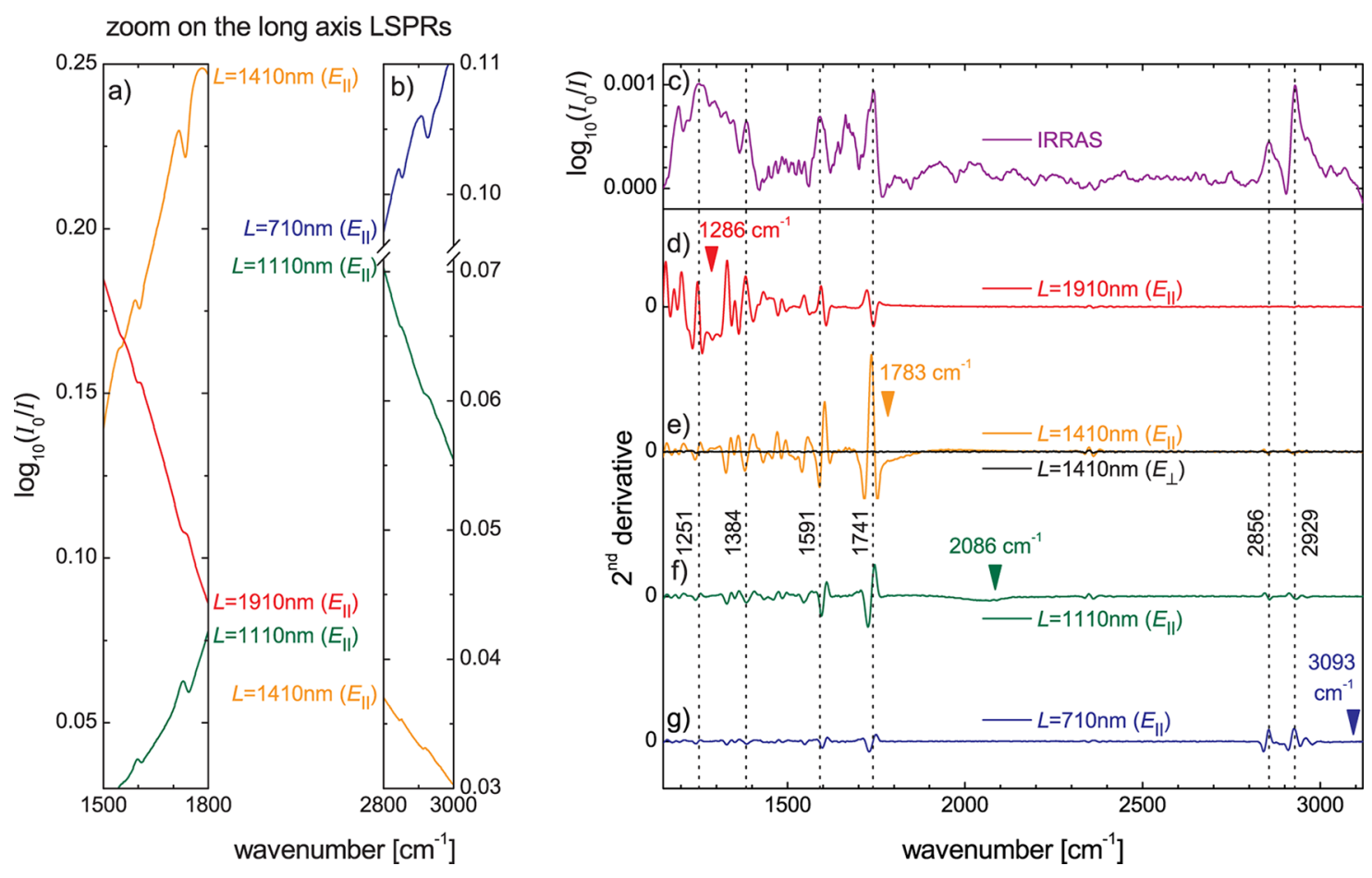

Figure 4. (a, b) Zoom-in of vibrational signals in IR extinction spectra from Figure 2 for the different antenna lengths (indicated). (c) Absorbance spectrum of a MB monolayer prepared on a gold mirror as calculated from IRRAS. The signals are close to the noise level and are disturbed by adsorption from water molecules in the IR beam path. Dashed lines indicate some of the major vibrational modes of MB. $(\mathbf{d}-\mathbf{g})$ Smoothed second derivative of the IR extinction spectra from Figure 2 for the different antenna lengths. All second derivatives use a common scale. The triangles indicate the positions of the localized plasmon resonance for each antenna array.

the second derivative of the Fano profile ${ }^{73}$ to the second derivative from experiment (see Supporting Information for more details) and reconstructed a background-free Fano profile (Figure S4). The peak size of this reconstruction is then compared with the reference, i.e., the unenhanced absorption signals from a monolayer. For such referencing, the signal of $M B$ in IRRAS was converted to an IR absorption signal; see Figure $4 \mathrm{C}$ and Figure $\mathrm{S} 5$ in the Supporting Information. For example, the antenna-enhanced IR active skeleton stretching vibration at $1591 \mathrm{~cm}^{-1}\left(I_{\text {SEIRS }}=5.2 \times 10^{-3}\right.$ abs. units in the background-free Fano profile) on the antenna (with $L=1410 \mathrm{~nm}$ ) is referenced to the same vibration on a planar substrate. The size of this reference signal is $I_{\text {ref }}=2.4 \times 10^{-5}$ abs. units. Furthermore, the different areas that contribute to the two kinds of signals (SEIRS and reference) have to be taken into account. For SEIRS that area is about $7200 \mathrm{~nm}^{2}$ per antenna, as indicated above. Within the measurement spot, which is much larger than the size of one antenna, each antenna occupies an area of about $7.4 \times 10^{6} \mathrm{~nm}^{2}$. So the ratio between the active area in SEIRS and the IR beam focus is 1:1000, whereas in IRRAS (or transmittance) this ratio is 1 for a homogeneous layer. For the same MB density on all kinds of gold surfaces, EF is 200000 in that case. Tuning and enhancement are even better for the vibration at $1741 \mathrm{~cm}^{-1}$, where the EF reaches 600000 (for $L=1410 \mathrm{~nm}$ ), as in other SEIRS studies. ${ }^{25,28}$ This EF represents a lower limit (see Methods section on reference samples for more details), and indeed, higher EF is possible. Longitudinal gaps below $50 \mathrm{~nm}$ increase near-field coupling of antennas, ${ }^{52}$ and, therefore, additional near-field confinement in the gaps may lead to higher EF. Furthermore, near-field coupling broadens the LSPR, ${ }^{52}$ which also broadens the spectral range for vibrational sensing. In this study, for certain antenna lengths (Figure $4 \mathrm{f}$ and $\mathrm{g}$ ), vibrational signals between 1500 and $3000 \mathrm{~cm}^{-1}$ are enhanced by 5 orders of magnitude. So, simple nanoantannas can provide multiband vibrational enhancement also in SEIRS.

\section{CONCLUSIONS}

SERS and SEIRS using the same linear optical antennas have been successfully demonstrated. SEIRS enhancement factors up to $6 \times 10^{5}$, comparable to former studies, is observed exciting the longitudinal dipolar resonance. SERS amplification between $10^{2}$ and $10^{3}$ (electromagnetic enhancement) is obtained exciting the transverse LSPR. While in the IR the enhanced fields are confined at the nanoantenna edges, the excitation of the transverse LSPR allows us to extend the enhanced near-field zone to all the molecules present over the antenna surface within the laser spot, permitting one to achieve SERS from a single nanoantenna. By changing the length of the 
antenna, multiple vibrational lines can be enhanced in SEIRS in an extended range up to $3000 \mathrm{~cm}^{-1}$, enabling improved identification of molecules and observation of molecular changes. Since IR and Raman scattering cross sections are different and since different selection rules hold for Raman and IR, doing SERS and SEIRS on the same plasmonic elements allows one to obtain complementary vibrational information. With further developments of integrated IR and Raman spectroscopic microscopy, the combination of SERS and SEIRS on the same single nanoantenna will become reality and provide unique advantages. The first benefit is miniaturization of the device: having a micrometerscale nanosensor would simplify the implementation on lab-on-a-chip architectures and trigger the development of ultracompact multiplex detection systems in which each nanoantenna is dedicated to the detection of a specific analyte. Reduction of the analyte is another benefit: a few nanoantennas (or ideally a single nanoantenna) integrated in a micro- or nanofluidic chip would prevent any spill out of the analyte on different plasmonic substrates or on zones of the substrate wider than the ones actually probed. Finally, the reproducibility of the sensor is another advantage: especially when quantitative measurements are needed, any signal fluctuation related to the inhomogeneous signal enhancement of different nanoantennas should be avoided.

From the nanoantenna side, a series of improvements can be easily envisioned to optimize the performances of our concept of a SERS-SEIRS nanosensor and to better fulfill potential applications. Indeed, higher SERS enhancement factors can be achieved with other materials (e.g., silver). SEIRS and SERS can be further optimized with a more complex structure of the plasmonic nanoparticle. Applications to a broader spectrum of nonresonant molecules could pave the way for high-sensitivity, quick, and safe identification of rare molecules in pollutant detection and of molecular biomarkers in medical early diagnosis. The ease of tuning both resonances of one simple shaped object makes our idea superior to previously published approaches.

\section{METHODS}

Gold Nanoantenna Fabrication. Electron beam lithography of the nanoantenna structures ${ }^{74}$ has been carried out on $\mathrm{CaF}_{2}$ (100) substrates spin-coated with polymethyl methacrylate resist (MicroChem 950 PMMA A3). A thin layer of aluminum is thermally evaporated on the PMMA surface in order to avoid charging effects. Electron exposure is performed at $20 \mathrm{keV}$ beam energy and $450 \mu \mathrm{C} / \mathrm{cm}^{2}$ exposure dose. After the $\mathrm{Al}$ removal in a $\mathrm{KOH}(1 \mathrm{M})$ solution, the exposed resist is developed in a conventional solution of MIBK/IPA (1:3) for $30 \mathrm{~s}$. Then, a $5 \mathrm{~nm}$ adhesion layer of $\mathrm{Ti}$ and a $55 \mathrm{~nm} \mathrm{Au}$ film are evaporated inside a high-vacuum Kurt J. Lesker thin-film vapor deposition system. The unexposed resist is removed with acetone and rinsed in isopropyl alcohol. $\mathrm{O}_{2}$ plasma washing at $200 \mathrm{~W}$ for $60 \mathrm{~s}$ is used to remove residual photoresist and organic contaminants.

Binding of Methylene Blue to Nanoantennas. Binding of $M B$ (purchased from Aldrich) is accomplished by immersing the samples in a solution of $\mathrm{MB}\left(10^{-4} \mathrm{M}\right)$ in deionized water for at least $1 \mathrm{~h}$, followed by immediate rinsing in ultrapure water to remove the $M B$ excess and drying in air. ${ }^{67,75} \mathrm{MB}$ is soluble in water, where it forms positive cations $\left(\mathrm{MB}^{+}\right)^{76}$ that adsorb on gold surfaces via $\mathrm{MB}^{+}-\mathrm{Au}$ interaction, yielding stable chemical bonds with a partial charge transfer with gold. ${ }^{77}$ Monomers, dimers, and/or small aggregates might be present on our samples. However, related to the differences in absorption behavior (Figure S1a), monomers and dimers can be distinguished from multimers and dominate absorption at $633 \mathrm{~nm}$ (more details on binding of MB to gold in the Supporting Information).

IR Extinction and SEIRS Spectroscopy. SEIRS measurements are carried out in transmission mode with a purged IR microscope (Bruker Hyperion 1000) coupled to an FTIR spectrometer (Bruker Tensor 27). This setup enables focusing the IR light on a spot of $58 \mu \mathrm{m}$ diameter, illuminating about $270,360,460$, or 690 nanoantennas of the respective arrays. The light is linearly polarized with the electrical field vector along the long axis of the antennas to excite the LSPR in the IR spectral range. We also took an IR spectrum of each array with perpendicular polarization, to check the signal of our sample without exciting the IR-LSPR. All IR spectra are acquired with a resolution of $2 \mathrm{~cm}^{-1}$ and normalized to a respective reference spectrum taken without the feature of interest. For the measurement of the nanoantennas, this is a spectrum of the same $\mathrm{CaF}_{2}$ substrate at a position next to the arrays.

Visible Range Extinction and SERS Spectroscopy. Extinction and SERS measurements are carried out on the same Horiba JobinYvon HR800 spectrometer coupled with an Olympus BX51 optical microscope. The extinction spectra are acquired in transmission geometry, exciting the sample with a white xenon lamp (embedded in the microscope) whose light is first polarized using a ThorLabs LPVIS100 linear polarizer, and then focused onto the sample with a condenser lens on a spot having a diameter of $\sim 100 \mu \mathrm{m}$ (several antennas are excited). The radiation transmitted by the nanoantenna samples is collected by a $50 \times$ long working distance objective (numerical aperture, NA $=0.5$ ), spectrally dispersed by a $600 \mathrm{I}$ / $\mathrm{mm}$ grating and acquired by a Peltier-cooled CCD. As a reference signal we use the light signal transmitted through the $\mathrm{CaF}_{2}$ substrate. The SERS spectra are acquired in a backscattering configuration using a HeNe laser $(\lambda=633 \mathrm{~nm})$ as light source. Using a $100 \times$ objective (NA $=0.9$ ) the laser beam is focused on a $\sim 600 \mathrm{~nm}$ diameter spot (the object pupil is not overfilled). Excitation powers vary from $500 \mathrm{nW}$ to $50 \mu \mathrm{W}$ on the sample. The backscattered light passes an edge filter to remove the elastic scattering (rejection ratio $>10^{7}$ ) and is then spectrally dispersed and detected as described above. To avoid laserinduced chemical modifications of the molecule, ${ }^{78}$ experimentally observable with the appearance of a more and more intense peak at $478 \mathrm{~cm}^{-1}$ with respect to the adjacent 446 and $501 \mathrm{~cm}^{-1}$ peaks and a simultaneous decrease of the overall signal intensity, we adjust the laser power $(0.5-50 \mu \mathrm{W})$ and the integration time $(30-60 \mathrm{~s})$ so that the $478 \mathrm{~cm}^{-1}$ peak is negligible and so that two subsequent spectra acquired on the same point of the sample do not show intensity variations larger than the noise level.

FDTD Simulations. Numerical calculations are carried out with a commercial-grade simulator (Lumerical 8.0.1), ${ }^{79}$ permitting us to model the real systems used for the experiments $\left(L=1410 \mathrm{~nm}, w=h=60 \mathrm{~nm}\right.$ gold antennas grown on $\mathrm{CaF}_{2}$ with a $5 \mathrm{~nm}$ Ti interlayer, as shown in Figure $1 \mathrm{a}$ and $\mathrm{b}$ ). In order to resolve the round ends of the structure, subgridding techniques are used to obtain mesh sizes down to $1 \times 1 \times 1 \mathrm{~nm}^{3}$. Outside the antennas, a mesh size of $15 \mathrm{~nm}$ for the transversal and $50 \mathrm{~nm}$ for the longitudinal excitation is chosen. The IR optical data are taken from Palik, ${ }^{80}$ and the data for the visible spectral 
range from Johnson and Christy. ${ }^{81}$ Convergence tests have been performed by varying the thickness of the perfectly matched layer (PML), the mesh accuracy, and the simulation time. In the IR, since the experimental illumination spot is much larger than the antennas, a broad band $\left(800-8000 \mathrm{~cm}^{-1}\right)$ plane wave with longitudinal polarization is used to model the incident field. Periodic boundary conditions are used in the in-plane longitudinal and transverse directions in order to simulate the experimental geometry of the antenna arrays. In the out-of-plane $z$-direction the finite volume is surrounded by 40 layers of PML and the antenna is placed at least one wavelength away from the PML. In the visible, a Gaussian beam with a radius (decay of the electromagnetic field to 1/e) of $300 \mathrm{~nm}$ (dashed circle in Figure 1d) and transversal polarization is used to model the excitation. Since the optical coupling between the antennas is very low for the transversal excitation, only three antennas are placed in the simulation volume, which was surrounded by 40 PMLs in all directions. The local field is evaluated in the plane $30 \mathrm{~nm}$ above the $\mathrm{CaF}_{2}$ surface, which intersects the antenna at half its height. See also Supporting Information for more details on the near-field representation and sites with highest confinement.

SERS and SEIRS Reference Samples and Measurements. Reference measurements to evaluate the SERS and SEIRS enhancement factors are taken on flat gold substrates on which $M B$ is adsorbed. The SERS reference signal is acquired on the gold patches (width $40 \times 10 \mu \mathrm{m}^{2}$, thickness $60 \mathrm{~nm}$, RMS roughness $1.1 \mathrm{~nm}$ ) present on the $\mathrm{CaF}_{2}$ substrate after lithographic processing of the antennas and used as markers to localize the nanoantenna arrays. MB binds to the gold patches during the immersion of the nanoantenna chip in the MB solution.

IR reflection absorption spectroscopy measurements (IRRAS, $83^{\circ}$ incidence, p-polarization, $2 \mathrm{~cm}^{-1}$ resolution) have been performed on a larger $(25 \mathrm{~mm} \times 25 \mathrm{~mm})$ gold mirror with similar RMS roughness to obtain an unenhanced IR fingerprint of the MB molecules, used as a SEIRS reference-data source. The gold mirror has been immersed in a $33 \mu \mathrm{M}$ aqueous solution of $\mathrm{MB}$ for $15 \mathrm{~h}$ and rinsed with ultrapure water afterward. The reference for IRRAS has been taken on the same gold mirror before immersing it in the aqueous MB solution. IRRAS measurements have been compared with IR spectra acquired on a dried drop of $\mathrm{MB}$ on $\mathrm{CaF}_{2}$ in order to verify IR molecular signals (Figure S5). In the latter case the samples are prepared as follows: to raise the signal above noise level, we increased the optical density of $M B$ on the transparent support by drop casting a $1 \mu \mathrm{L}$ drop of $33 \mu \mathrm{M} \mathrm{MB}$ solution in ultrapure water on the bare $\mathrm{CaF}_{2}$ substrate. After $2 \mathrm{~h}$ all water has evaporated, leaving a MB stain on the surface. For IRRAS, the presence of $u \mathrm{~m}$ sized MB agglomerates on the $25 \times 25 \mathrm{~mm}^{2}$ gold surface cannot be excluded. Any agglomerates may have increased the reference signal and thus lowered the calculated SEIRS enhancement factor. So, real enhancement may be bigger than the calculated one.

Conflict of Interest: The authors declare no competing financial interest.

Acknowledgment. We acknowledge support from the European Union Seventh Framework Programme (FP7/20072013) under Grant Agreement No. 241818 (FP7-HEALTHF5-2009-241818-NANOANTENNA). B.F., C.d'A., E.M., O.M.M., and P.G.G. acknowledge support by MIUR under Project PRIN 2008J858Y7 and by Programma Operativo Nazionale Ricerca e Competitività 2007-2013, PON01 01322 PANREX. J.B. acknowledges support from the Heidelberg Graduate School of Fundamental Physics.

Supporting Information Available: UV-vis spectra of MB in liquid and of a dry drop, multiwavelength excitation SERS, further information on MB binding to gold substrates, microRaman spectra of MB in liquid, IRRAS and IR spectra on a dry drop of MB, details on FDTD simulations of near fields, and mathematical SERS/SEIRS enhancement evaluation. This material is available free of charge via the Internet at http:// pubs.acs.org.

\section{REFERENCES AND NOTES}

1. Novotny, L.; Van Hulst, N. Antennas for Light. Nat. Photonics 2011, 5, 83-90.

2. Mühlschlegel, P.; Eisler, H.-J.; Martin, O. J. F.; Hecht, B.; Pohl, D. W. Resonant Optical Antennas. Science (New York, N.Y.) 2005, 308, 1607-1609.

3. Aizpurua, J.; Bryant, G. W.; Richter, L. J.; Garcia de Abajo, F. J. Optical Properties of Coupled Metallic Nanorods for FieldEnhanced Spectroscopy. Phys. Rev. B 2005, 71, 235420.

4. Halas, N. J.; Lal, S.; Chang, W.-S.; Link, S.; Nordlander, P. Plasmons in Strongly Coupled Metallic Nanostructures. Chem. Rev. 2011, 111, 3913-3961.

5. Wei, H.; Reyes-Coronado, A.; Nordlander, P.; Aizpurua, J.; $\mathrm{Xu}, \mathrm{H}$. Multipolar Plasmon Resonances in Individual Ag Nanorice. ACS Nano 2010, 4, 2649-2654.

6. Dorfmuller, J.; Vogelgesang, R.; Weitz, R. T.; Rockstuhl, C.; Etrich, C.; Pertsch, T.; Lederer, F.; Kern, K. Fabry-Perot Resonances in One-Dimensional Plasmonic Nanostructures. Nano Lett. 2009, 9, 2372-2377.

7. Stockman, M. Nanofocusing of Optical Energy in Tapered Plasmonic Waveguides. Phys. Rev. Lett. 2004, 93, 137404.

8. Nordlander, P.; Oubre, C.; Prodan, E.; Li, K.; Stockman, M. I. Plasmon Hybridization in Nanoparticle Dimers. Nano Lett. 2004, 4, 899-903.

9. Kim, W.; Safonov, V.; Shalaev, V.; Armstrong, R. Fractals in Microcavities: Giant Coupled, Multiplicative Enhancement of Optical Responses. Phys. Rev. Lett. 1999, 82, 4811-4814.

10. Meier, S. A. Plasmonics: Fundamentals and Applications; Springer: New York, 2007.

11. Zayatsa, A. V.; Smolyaninov, I. I.; Maradudin, A. A. Nanooptics of Surface Plasmon Polaritons. Phys. Rep. 2005, 408, 131-314.

12. Moskovitz, M. Surface-Enhanced Spectroscopy. Rev. Mod. Phys. 1985, 57, 783-826.

13. Kneipp, K.; Moskovits, M.; Kneipp, H. Surface Enhanced Raman Scattering; Springer: New York, 2006.

14. Le Ru, E.; Etchegoin, P. Principles of Surface Enhanced Raman Spectroscopy; Elsevier: Amsterdam, 2009.

15. Le Ru, E. C.; Blackie, E.; Meyer, M.; Etchegoin, P. G. Surface Enhanced Raman Scattering Enhancement Factors: A Comprehensive Study. J. Phys. Chem. C 2007, 111, 13794-13803.

16. Ward, D. R.; Halas, N. J.; Ciszek, J. W.; Tour, J. M.; Wu, Y.; Nordlander, P.; Natelson, D. Simultaneous Measurements of Electronic Conduction and Raman Response in Molecular Junctions. Nano Lett. 2008, 8, 919-924.

17. Etchegoin, P. G.; Le Ru, E. C.; Fainstein, A. Bi-Analyte Single Molecule SERS Technique with Simultaneous Spatial Resolution. Phys. Chem. Chem. Phys. 2011, 13, 4500-4506.

18. Hartstein, A.; Kirtley, R. J.; Tsang, C. J. Enhancement of the Infrared Absorption from Molecular Monolayers with Thin Metal Overlayers. Phys. Rev. Lett. 1980, 45, 201-204.

19. Wang, H.; Kundu, J.; Halas, N. J. Plasmonic Nanoshell Arrays Combine Surface-Enhanced Vibrational Spectroscopies on a Single Substrate. Angew. Chem., Int. Ed. 2007, 46, 9040-9044.

20. Osawa, M.; Ikeda, M. Surface-Enhanced Infrared Absorption of p-Nitrobenzoic Acid Deposited on Silver Island Films: Contributions of Electromagnetic and Chemical Mechanisms. J. Phys. Chem. 1991, 95, 9914-9919.

21. Enders, D.; Pucci, A. Surface Enhanced Infrared Absorption of Octadecanethiol on Wet-Chemically Prepared Au Nanoparticle Films. Appl. Phys. Lett. 2006, 88, 184104.

22. Aroca, R. F.; Ross, D. J.; Domingo, C. Surface-Enhanced Infrared Spectroscopy. Appl. Spectrosc. 2004, 58, 324A338A.

23. Adato, R.; Yanik, A. A.; Altug, H. On Chip Plasmonic Monopole Nano-Antennas. Nano Lett. 2011, 11, 5219-5226.

24. Chen, K.; Adato, R.; Altug, H. Dual-Band Perfect Absorber for Multispectral Plasmon-Enhanced Infrared Spectroscopy. ACS Nano 2012, 6, 7998-8006.

25. Neubrech, F.; Pucci, A.; Cornelius, T.; Karim, S.; GarcíaEtxarri, A.; Aizpurua, J. Resonant Plasmonic and Vibrational Coupling in a Tailored Nanoantenna for Infrared Detection. Phys. Rev. Lett. 2008, 101, 157403. 
26. Liberman, V.; Adato, R.; Jeys, T. H.; Saar, B. G.; Erramilli, S.; Altug, H. Rational Design and Optimization of Plasmonic Nanoarrays for Surface Enhanced Infrared Spectroscopy. Opt. Express 2012, 20, 11953-11966.

27. Alonso-Gonzalez, P.; Albella, P.; Schnell, M.; Chen, J.; Huth, F.; Garcia-Etxarri, A.; Casanova, F.; Golmar, F.; Arzubiaga, L.; Hueso, L. E.; et al. Resolving the Electromagnetic Mechanism of Surface-Enhanced Light Scattering at Single Hot Spots. Nat. Commun. 2012, 3, 684.

28. Neubrech, F.; Pucci, A. Plasmonic Enhancement of Vibrational Excitations in the Infrared. IEEE J. Sel. Top. Quantum Electron. 2012, DOI: 10.1109/JSTQE.2012.2227302.

29. Cataldo, S.; Zhao, J.; Neubrech, F.; Frank, B .; Zhang, C.; Braun, P. V.; Giessen, H. Hole-Mask Colloidal Nanolithography for Large-Area Low-Cost Metamaterials and AntennaAssisted Surface-Enhanced Infrared Absorption Substrates. ACS Nano 2012, 6, 979-985.

30. Adato, R.; Yanik, A. A.; Amsden, J. J.; Kaplan, D. L.; Omenetto, F. G.; Hong, M. K.; Erramilli, S.; Altug, H. Ultra-Sensitive Vibrational Spectroscopy of Protein Monolayers with Plasmonic Nanoantenna Arrays. Proc. Natl. Acad. Sci. U.S.A 2009, 106, 19227-19232.

31. Bishop, D. M. Group Theory and Chemistry; Dover: Mineola, 1993.

32. Kellner, R.; Lendl, B.; Wells, I.; Worsfold, P. J. Comparison of Univariate and Multivariate Strategies for the Determination of Sucrose in Fruit Juices by Automated Flow Injection Analysis with Fourier Transform Infrared Detection. Appl. Spectrosc. 1997, 51), 68A-80A.

33. David, C.; D'Andrea, C.; Lancelot, E.; Bochterle, J.; Guillot, N.; Fazio, B.; Maragò, O. M.; Sutton, A.; Charnaux, N.; Neubrech, F.; et al. Raman and IR Spectroscopy of Manganese Superoxide Dismutase, a Pathology Biomarker. Vib. Spectrosc. 2012, 62, 50-58.

34. Rubim, J. C.; Trindade, F. A.; Gelesky, M. A.; Aroca, R. F.; Dupont, J. Surface-Enhanced Vibrational Spectroscopy of Tetrafluoroborate 1-n-Butyl-3-methylimidazolium (BMIBF $\left.{ }_{4}\right)$ Ionic Liquid on Silver Surfaces. J. Phys. Chem. C 2008, 112, 19670-19675.

35. Kudelski, A. Characterization of Thiolate-Based Mono- and Bilayers by Vibrational Spectroscopy: A Review. Vib. Spectrosc. 2005, 39, 200-213.

36. Zhang, Z.; Imae, T.; Sato, H.; Watanabe, A.; Ozaki, Y. SurfaceEnhanced Raman Scattering and Surface-Enhanced Infrared Absorption Spectroscopic Studies of a Metalloporphyrin Monolayer Film Formed on Pyridine SelfAssembled Monolayer-Modified Gold. Langmuir 2001, 17, 4564-4568.

37. Alvarez-Puebla, R. A.; Garrido, J. J.; Aroca, R. F. SurfaceEnhanced Vibrational Microspectroscopy of Fulvic Acid Micelles. Anal. Chem. 2004, 76, 7118-7125

38. Kundu, J.; Neumann, O.; Janesko, B. G.; Zhang, D.; Lal, S.; Barhoumi, A.; Scuseria, G. E.; Halas, N. J. Adenine- and Adenosine Monophosphate (AMP)-Gold Binding Interactions Studied by Surface-Enhanced Raman and Infrared Spectroscopies. J. Phys. Chem. C 2009, 113, 14390-14397.

39. Smith, M.; Stambaugh, K.; Smith, L.; Son, H.-J.; Gardner, A.; Cordova, S.; Posey, K.; Perry, D.; Biris, A. S. Surface-Enhanced Vibrational Investigation of Adsorbed Analgesics. Vib. Spectrosc. 2009, 49, 288-297.

40. Levin, C. S.; Kundu, J.; Janesko, B. G.; Scuseria, G. E.; Raphael, R. M.; Halas, N. J. Interactions of Ibuprofen with Hybrid Lipid Bilayers Probed by Complementary SurfaceEnhanced Vibrational Spectroscopies. J. Phys. Chem. B 2008, 112, 14168-14175.

41. Le, F.; Brandl, D. W.; Urzhumov, Y. A.; Wang, H.; Kundu, J.; Halas, N. J.; Aizpurua, J.; Nordlander, P. Metallic Nanoparticle Arrays: A Common Substrate for Both SurfaceEnhanced Raman Scattering and Surface-Enhanced Infrared Absorption. ACS Nano 2008, 2, 707-718.

42. Lal, S.; Grady, N. K.; Kundu, J.; Levin, C. S.; Lassiter, J. B.; Halas, N. J. Tailoring Plasmonic Substrates for Surface Enhanced Spectroscopies. Chem. Soc. Rev. 2008, 37, 898-911.

43. Baia, M.; Toderas, F.; Baia, L.; Maniu, D.; Astilean, S. Multilayer Structures of Self-Assembled Gold Nanoparticles as a
Unique SERS and SEIRA Substrate. Chem. Phys. Chem 2009, 10, 1106-1111.

44. Fasasi, A.; Griffiths, P. R.; Pan, H. B.; Wai, C. M. SurfaceEnhanced Vibrational Spectroscopy of Adsorbates on Microemulsion Synthesized Gold Nanoparticles. Appl. Spectrosc. 2011, 65), 741-745.

45. Domingo, C.; Resta, V.; Sanchez-Cortes, S.; Garcia-Ramos, J. V.; Gonzalo, J. Pulsed Laser Deposited Au Nanoparticles as Substrates for Surface-Enhanced Vibrational Spectroscopy. J. Phys. Chem. C 2007, 111, 8149-8152.

46. Delgado, J. M.; Orts, J. M.; Pérez, J. M.; Rodes, A. Sputtered Thin-Film Gold Electrodes for in Situ ATR-SEIRAS and SERS Studies. J. Electroanal. Chem. 2008, 617, 130-140.

47. Leverette, C. L.; Jacobs, S. A.; Shanmukh, S.; Chaney, S. B.; Dluhy, R. A.; Zhao, Y. P. Aligned Silver Nanorod Arrays as Substrates for Surface-Enhanced Infrared Absorption Spectroscopy. Appl. Spectrosc. 2006, 60, 906-913.

48. Prokopec, V.; Dendisová-Vyškovská, M.; Kokaislová, A.; Čejková, J.; Člupek, M.; Matějka, P. Spectroscopic Study of SERS- and SEIRA-Activity of Copper Large-Scaled Surface Substrates Prepared by Electrochemical Deposition: What Is the Role of Oxidation-Reduction Cycle Treatment? J. Mol. Struct. 2011, 993, 410-419.

49. Huang, B.-B.; Wang, J.-Y.; Huo, S.-J.; Cai, W.-B. Facile Fabrication of Silver Nanoparticles on Silicon for Surface-Enhanced Infrared and Raman Analysis. Surf. Interface Anal. 2008, 40, 81-84.

50. Grand, J.; Lamy de la Chapelle, M.; Bijeon, J.-L.; Adam, P.-M.; Vial, A.; Royer, P. Role of Localized Surface Plasmons in Surface-Enhanced Raman Scattering of Shape-Controlled Metallic Particles in Regular Arrays. Phys. Rev. B 2005, 72, 033407.

51. Félidj, N.; Aubard, J.; Lévi, G.; Krenn, J. R.; Salerno, M.; Schider, G.; Lamprecht, B.; Leitner, A.; Aussenegg, F. R. Controlling the Optical Response of Regular Arrays of Gold Particles for Surface-Enhanced Raman Scattering. Phys. Rev. B 2002, 65, 075419.

52. Weber, D.; Albella, P.; Alonso-González, P.; Neubrech, F.; Gui, H.; Nagao, T.; Hillenbrand, R.; Aizpurua, J.; Pucci, A. Longitudinal and Transverse Coupling in Infrared Gold Nanoantenna Arrays: Long Range versus Short Range Interaction Regimes. Opt. Express 2011, 19, 15047-15061.

53. McFarland, A. D.; Young, M.; Dieringer, J.; Van Duyne, R. P. Wavelength-Scanned Surface-Enhanced Raman Excitation Spectroscopy. J. Phys. Chem. B 2005, 109, 11279-11285.

54. Guillot, N.; Shen, H.; Frémaux, B.; Péron, O.; Rinnert, E.; Toury, T.; Lamy de la Chapelle, M. Surface Enhanced Raman Scattering Optimization of Gold Nanocylinder Arrays: Influence of the Localized Surface Plasmon Resonance and Excitation Wavelength. Appl. Phys. Lett. 2010, 97, 023113.

55. Anderson, L. J. E.; Payne, C. M.; Zhen, Y.-R.; Nordlander, P.; Hafner, J. H. A Tunable Plasmon Resonance in Gold Nanobelts. Nano Lett. 2011, 11, 5034-5037.

56. Xiao, Y.; Lai, R. Y.; Plaxco, K. W. Preparation of ElectrodeImmobilized, Redox-Modified Oligonucleotides for Electrochemical DNA and Aptamer-Based Sensing. Nat. Protoc. 2007, 2, 2875-2880.

57. Jockush, S.; Lee, D.; Turro, N. J.; Leonard, E. F. PhotoInduced Inactivation of Viruses: Adsorption of Methylene Blue, Thionine, and Thiopyronine on Qbeta Bacteriophage. Proc. Natl. Acad. Sci. U.S.A. 1996, 93, 7446.

58. Laurent, G.; Félidj, N.; Lau Truong, S.; Aubard, J.; Lévi, G.; Krenn, J. R.; Hohenau, A.; Leitner, A.; Aussenegg, F. R. Imaging Surface Plasmon of Gold Nanoparticle Arrays by Far-Field Raman Scattering. Nano Lett. 2005, 5, 253-258.

59. Fazio, B.; D'Andrea, C.; Bonaccorso, F.; Irrera, A.; Calogero, G.; Vasi, C.; Gucciardi, P. G.; Allegrini, M.; Toma, A.; Chiappe, D.; et al. Re-Radiation Enhancement in Polarized SurfaceEnhanced Resonant Raman Scattering of Randomly Oriented Molecules on Self-Organized Gold Nanowires. ACS Nano 2011, 5, 5945-5956.

60. Jockush, S.; Turro, N. J.; Tomalia, D. A. Aggregation of Methylene Blue on Starbust Dendrimers. Macromolecules 1995, 28, 7416-7418. 
61. Nicolai, S. H. A.; Rubim, J. C. Surface-Enhanced Resonance Raman (SERR) Spectra of Methylene Blue Adsorbed on a Silver Electrode. Langmuir 2003, 19, 4291-4294.

62. Le Ru, E. C.; Meyer, S. A.; Artur, C.; Etchegoin, P. G.; Grand, J.; Lang, P.; Maurel, F. Experimental Demonstration of Surface Selection Rules for SERS on Flat Metallic Surfaces. Chem. Commun. 2011, 47, 3903-3905.

63. Naujok, R. R.; Duevel, R. V.; Corn, R. M. Fluorescence and Fourier Transform Surface-Enhanced Raman Scattering Measurements of Methylene Blue Adsorbed onto a SulfurModified Gold Electrode. Langmuir 1993, 9, 1771-1774.

64. Yu, Z.; Chuang, S. S. C. Probing Methylene Blue Photocatalytic Degradation by Adsorbed Ethanol with in Situ IR. J. Phys. Chem. C 2007, 111, 13813-13820.

65. Neubrech, F.; Kolb, T.; Lovrincic, R.; Fahsold, G.; Pucci, A.; Aizpurua, J.; Cornelius, T. W.; Toimil-Molares, M. E.; Neumann, R.; Karim, S. Resonances of Individual Metal Nanowires in the Infrared. Appl. Phys. Lett. 2006, 89, 253104.

66. Grand, J.; Adam, P.-M.; Grimault, A.-S.; Vial, A.; Lamy de la Chapelle, M.; Bijeon, J.-L.; Kostcheev, S.; Royer, P. Optical Extinction Spectroscopy of Oblate, Prolate and Ellipsoid Shaped Gold Nanoparticles: Experiments and Theory. Plasmonics 2006, 1, 135-140.

67. Laurent, G.; Felidj, N.; Aubard, J.; Levi, G.; Krenn, J. R.; Hohenau, A.; Schider, G.; Leitner, A.; Aussenegg, F. R. Evidence of Multipolar Excitations in Surface Enhanced Raman Scattering. Phys. Rev. B 2005, 71, 045430.

68. Billot, L.; Lamy de la Chapelle, M.; Grimault, A.-S.; Vial, A.; Barchiesi, D.; Bijeon, J.-L.; Adam, P.-M.; Royer, P. Surface Enhanced Raman Scattering on Gold Nanowire Arrays: Evidence of Strong Multipolar Surface Plasmon Resonance Enhancement. Chem. Phys. Lett. 2006, 422, 303-307.

69. Tognalli, N. G.; Fainstein, A.; Vericat, C.; Vela, M. E.; Salvarezza, R. C. In Situ Raman Spectroscopy of Redox Species Confined in Self-Assembled Molecular Films. J. Phys. Chem. C 2008, 112, 3741-3746.

70. Zuloaga, J.; Nodlander, P. On the Energy Shift between Near-Field and Far-Field Peak Intensities in Localized Plasmon Systems. Nano Lett. 2011, 11, 1280-1283.

71. Giannini, V.; Francescato, Y.; Amrania, H.; Phillips, C. C.; Maier, S. A. Fano Resonances in Nanoscale Plasmonic Systems: A Parameter-Free Modeling Approach. Nano Lett. 2011, 11, 2835-2840.

72. Maddams, W. F.; Tooke, P. B. Quantitative Conformational Studies on Poly(vinyl chloride). J. Macromol. Sci.: Part A : Chem. 1982, 17, 951-968.

73. Fano, U. Effects of Configuration Interaction on Intensities and Phase Shifts. Phys. Rev. 1961, 124, 1866-1878.

74. Neubrech, F.; Weber, D.; Katzmann, J.; Huck, Ch.; Toma, A.; Di Fabrizio, E.; Pucci, A.; Härtling, T. Infrared Optical Properties of Nanoantenna Dimers with Photochemically Narrowed Gaps in the $5 \mathrm{~nm}$ Regime. ACS Nano 2012, 6, 73267332.

75. Xiao, G.-N.; Man, S.-Q. Surface-Enhanced Raman Scattering of Methylene Blue Adsorbed on Cap-Shaped Silver Nanoparticles. Chem. Phys. Lett. 2007, 447, 305-309.

76. Tognalli, N. G.; Fainstein, A.; Vericat, C .; Vela, M. E.; Salvarezza, R. C. Exploring Three-Dimensional Nanosystems with Raman Spectroscopy: Methylene Blue Adsorbed on Thiol and Sulfur Monolayers on Gold. J. Phys. Chem. B 2006, 110, 354-360.

77. Zutic, V.; Svetlicic, V.; Clavielier, J.; Chevalet, J. Supramolecular Phenomena in Organic Redox Films at Electrodes. J. Electroanal. Chem. 1987, 219, 183-195.

78. Lowe, R. D.; Snook, R. D. Photobleaching of Methylene Blue in Continuous Wave Thermal Lens Spectrometry. Analyst 1993, 118, 613-616.

79. Lumerical Solutions Inc. FDTD Solutions version 8.0.1. http://www.lumerical.com/tcad-products/fdtd/

80. Palik, E. D. Handbook of Optical Constants of Solids; Academic Press: New York, 1985.

81. Johnson, P. B.; Christy, R. W. Optical Constants of the Noble Metals. Phys. Rev. B 1972, 6, 4370-4379. 